\title{
Impact of corticosteroid treatment on clinical outcomes of influenza-associated ARDS: a nationwide multicenter study
}

Ming-Ju Tsai 1,2,3,4 , Kuang-Yao Yang ${ }^{5,6}$, Ming-Cheng Chan ${ }^{7,8,9}$, Kuo-Chin Kao ${ }^{10,11}$, Hao-Chien Wang ${ }^{12}$, Wann-Cherng Perng ${ }^{13}$, Chieh-Liang W $\mathrm{u}^{7,14}$, Shinn-Jye Liang ${ }^{15}$, Wen-Feng Fang ${ }^{16,17}$, Jong-Rung Tsai ${ }^{1,2,4}$ (D) , Wei-An Chang 1,2,3 (10, Ying-Chun Chien ${ }^{12}$, Wei-Chih Chen ${ }^{5,18}$, Han-Chung Hu ${ }^{10,11}$, Chiung-Yu Lin ${ }^{16}$, Wen-Cheng Chao ${ }^{7,19}$, Chau-Chyun Sheu ${ }^{1,2,3,4^{*}}$ (D) and for Taiwan Severe Influenza Research Consortium (TSIRC) Investigators

\begin{abstract}
Background: Corticosteroid treatment has been widely used in the treatment of septic shock, influenza, and ARDS, although some previous studies discourage its use in severe influenza patients. This multicenter retrospective cohort study conducted in the intensive care units (ICUs) of eight medical centers across Taiwan aims to determine the real-world status of corticosteroid treatment in patients with influenza-associated acute respiratory distress syndrome (ARDS) and its impact on clinical outcomes. Between October 2015 and March 2016, consecutive ICU patients with virology-proven influenza infections who fulfilled ARDS and received invasive mechanical ventilation were enrolled. The impact of early corticosteroid treatment ( $\geq 200 \mathrm{mg}$ hydrocortisone equivalent dose within 3 days after ICU admission, determined by a sensitivity analysis) on hospital mortality (the primary outcome) was assessed by multivariable logistic regression analysis, and further confirmed in a propensity score-matched cohort.
\end{abstract}

Results: Among the 241 patients with influenza-associated ARDS, 85 (35.3\%) patients receiving early corticosteroid treatment had similar baseline characteristics, but a significantly higher hospital mortality rate than those without early corticosteroid treatment [43.5\% (37/85) vs. 19.2\% (30/156), $p<0.001]$. Early corticosteroid treatment was independently associated with increased hospital mortality in overall patients [adjusted odds ratio $(95 \% \mathrm{Cl})=5.02$ (2.39-10.54), $p<0.001]$ and in all subgroups. Earlier treatment and higher dosing were associated with higher hospital mortality. Early corticosteroid treatment was associated with a significantly increased odds of subsequent bacteremia [adjusted odds ratio $(95 \% \mathrm{Cl})=2.37(1.01-5.56)]$. The analyses using a propensity score-matched cohort showed consistent results.

Conclusions: Early corticosteroid treatment was associated with a significantly increased hospital mortality in adult patients with influenza-associated ARDS. Earlier treatment and higher dosing were associated with higher hospital mortality. Clinicians should be cautious while using corticosteroid treatment in this patient group.

Keywords: Influenza, Steroid, Glucocorticoid, Acute respiratory distress syndrome, Mortality, Pneumonia

*Correspondence: sheucc@gmail.com

${ }^{1}$ Division of Pulmonary and Critical Care Medicine, Department of Internal Medicine, Kaohsiung Medical University Hospital, Kaohsiung Medical University, No. 100, Tz-You 1st Road, Kaohsiung 807, Taiwan Full list of author information is available at the end of the article

\section{Background}

Severe influenza is usually associated with high viral load and hypercytokinemia [1-4]. Based on the theoretical benefit in modulating hypercytokinemia and the clinical benefits shown in septic shock, corticosteroids

\section{Springer Open}

(c) The Author(s) 2020. This article is licensed under a Creative Commons Attribution 4.0 International License, which permits use, sharing, adaptation, distribution and reproduction in any medium or format, as long as you give appropriate credit to the original author(s) and the source, provide a link to the Creative Commons licence, and indicate if changes were made. The images or other third party material in this article are included in the article's Creative Commons licence, unless indicated otherwise in a credit line to the material. If material is not included in the article's Creative Commons licence and your intended use is not permitted by statutory regulation or exceeds the permitted use, you will need to obtain permission directly from the copyright holder. To view a copy of this licence, visit http://creativeco mmons.org/licenses/by/4.0/. 
have sometimes been used in severe influenza and influenza-associated acute respiratory distress syndrome (ARDS) [5, 6]. An European retrospective analysis of 208 patients with ARDS related to 2009 pandemic influenza A (H1N1), with $39.9 \%$ of patients receiving corticosteroid treatment, refuted the beneficial effects of corticosteroids for patients with influenza-associated ARDS and even showed an increased mortality associated with early corticosteroid treatment (within 3 days of mechanical ventilation) [7]. Another study of 2009 pandemic influenza A (H1N1)-related critical illness in Canada, with $46.1 \%$ of patients received corticosteroids, also revealed higher hospital mortality, ventilator days, and intensive care unit (ICU) days in patients using corticosteroids, while the odds ratio of association between corticosteroid treatment and hospital mortality decreased from 1.85 in the multivariable model to 0.96 after adjusting for time-dependent between-group differences using marginal structural modeling [8]. A Chinese study of avian influenza A (H7N9) viral pneumonia revealed a significantly higher 60 -day mortality in patients receiving corticosteroids, and high-dose corticosteroid treatment was significantly associated with higher 30-day and 60-day mortalities [9]. A systematic review and meta-analysis including 9 cohort studies and 14 case-control studies also showed that corticosteroid treatment was associated with higher mortality in patients with influenza A (H1N1) infection [10]. The secondary analysis of a Spanish multicenter prospective cohort study showed that corticosteroid therapy was associated with increased ICU mortality in patients with severe influenza [11]. Because corticosteroids might be more frequently and/or earlier used in sicker patients, a solid conclusion about the association between corticosteroid treatments and mortality in severe influenza could not be made.

Corticosteroids might theoretically dampen both inflammation and fibrosis, the cardinal mechanisms for lung injury and adverse outcomes in ARDS, but the use of corticosteroids in ARDS remained controversial despite being extensively evaluated in a plethora of studies [12, 13]. Earlier studies using high-dose corticosteroids showed no survival benefit, whereas using low-dose corticosteroids appeared to improve survival [14]. The timing also matters, while starting methylprednisolone more than 14 days after ARDS onset significantly increased mortality [15]. A systematic review and metaanalysis including 8 randomized controlled trials (RCTs) and 10 cohort studies revealed heterogeneity in effects of corticosteroids on ARDS, which might be influenced by the duration of outcome measures and the etiologies of ARDS [5]. This meta-analysis showed that corticosteroid treatment was not associated with better long-term outcomes in ARDS, and moreover, was associated with increased risk of mortality in influenza-related ARDS [5].

Although the role of corticosteroid treatment in ARDS and influenza has been challenged, this treatment is still frequently endorsed by clinicians for patients with influenza-associated ARDS [9, 13]. A large international multicenter prospective observational cohort study across 50 countries showed that $17.9 \%$ of ARDS patients received high-dose corticosteroids $(\geq 1 \mathrm{mg} / \mathrm{kg}$ methylprednisolone equivalent dose) [16]. In a Chinese study of influenza pneumonia, $70.8 \%$ of patients received corticosteroids [9].

The aims of this study were to understand the realworld condition of corticosteroid treatment in influenza-associated ARDS, and to investigate the impact of corticosteroid treatment on clinical outcomes in patients with influenza-associated ARDS.

\section{Methods}

\section{Study cohort}

This multicenter retrospective cohort study was conducted in the ICUs in eight medical centers across Taiwan during the 2015-2016 influenza epidemic [17-19]. The study was approved by the Institutional Review Board in each participating hospital.

All patients admitted to the ICUs between October 1, 2015 and March 31, 2016 with a diagnosis of virologyproven influenza infections were screened, and patients fulfilled the ARDS criteria and received invasive mechanical ventilation were enrolled in the study. The diagnosis of influenza was confirmed by the Taiwan Centers for Disease Control based on the rapid influenza diagnostic test, reverse transcription-polymerase chain reaction, or viral culture. The diagnosis and grading of ARDS was determined according to the Berlin definition [20].

\section{Measures}

Medical records of the eligible patients were reviewed and data were collected with a standardized case report form in the participating ICUs. The relevant information, including demographics, comorbidities, laboratory tests, influenza type, severity score, and specific treatments, were collected. The calendar day of ICU admission was defined as the first ICU day (ICU Day 1). The details of corticosteroid use (including the timing of initiation, dosing, and type of medications) were recorded, and the doses were converted to hydrocortisone equivalent doses. The cumulative corticosteroid dose was calculated from the ICU admission to the end of the specified day after ICU admission. Bacterial co-infections were defined as positive bacterial cultures from blood, pleural effusion, lower respiratory tract secretion, or urine samples within 
$48 \mathrm{~h}$ of ARDS diagnosis. Hospital mortality was taken as the primary outcome in this study.

\section{Statistical analysis}

Data are presented in number (\%) or median (25th-75th percentiles), as appropriate. Categorical variables and continuous variables were compared using $X^{2}$ test and Mann-Whitney $U$ test, respectively. Survival times were estimated using the Kaplan-Meier method, with differences between the groups compared using log-rank test. Cox proportional hazards regression analysis was used to identify the effects of variables on survival. Logistic regression analysis was used to identify the effects of variables on hospital mortality. Variables with a $p<0.05$ in univariate models were selected into the multivariable model, using a stepwise algorithm with criteria of $p>0.05$ for eliminating variables. Missing values were replaced by the corresponding overall median values for multivariable analyses.

To account for residual confounding by indication of the associations between early corticosteroid treatment and clinical outcomes, variables potentially associated with early corticosteroid treatment, including age, sex, Acute Physiology and Chronic Health Evaluation (APACHE) II score, influenza type, ARDS severity, bacterial coinfection, vasopressor infusion, prone positioning, extracorporeal membrane oxygenation (ECMO) treatment, and chronic airway disease, were included in a logistic regression model with early corticosteroid treatment as the dependent variable to determine a propensity score for treatment. A propensity score-matched cohort was selected from the original cohort and similar analyses were performed to confirm our findings from the original cohort.

All statistical analyses were performed using SAS system (version 9.4 for Windows, SAS Institute Inc., Cary, NC, USA). The statistical significance level was set at a two-sided $p$ value of $<0.05$. A hazard ratio (HR) or an odds ratio (OR) was reported along with $95 \%$ confidence interval $(\mathrm{CI})$.

\section{Results}

\section{Analyses of the whole study population}

Totally, 336 patients admitted to the ICUs with virologyproven complicated influenza were screened (Additional file 1: Fig. S1). After excluding 54 patients without ARDS, 35 patients who did not receive mechanical ventilation, and 6 patients with incomplete data, the data of the remaining 241 patients, including 174 (72.2\%) survivors and $67(27.8 \%)$ non-survivors, were used for analyses (Additional file 1: Table S1).

A substantial proportion of patients with influenzaassociated ARDS in our study population had received corticosteroid treatment during their ICU stay. By the end of the third ICU day, $35.3 \%$ of patients had received $\geq 200 \mathrm{mg}$ hydrocortisone equivalent dose, and the proportion increased to $57.7 \%$ by the end of the second week after ICU admission (Additional file 1: Fig. S2).

We performed a sensitivity analysis, which used univariate logistic regression analyses to assess the effects of various cumulative doses of corticosteroids by various ICU days on the hospital mortality (Fig. 1). Corticosteroid treatment was significantly associated with hospital mortality for all proposed criteria, while there was a trend that the earlier use of corticosteroids was associated with the higher odds of hospital mortality in patients with

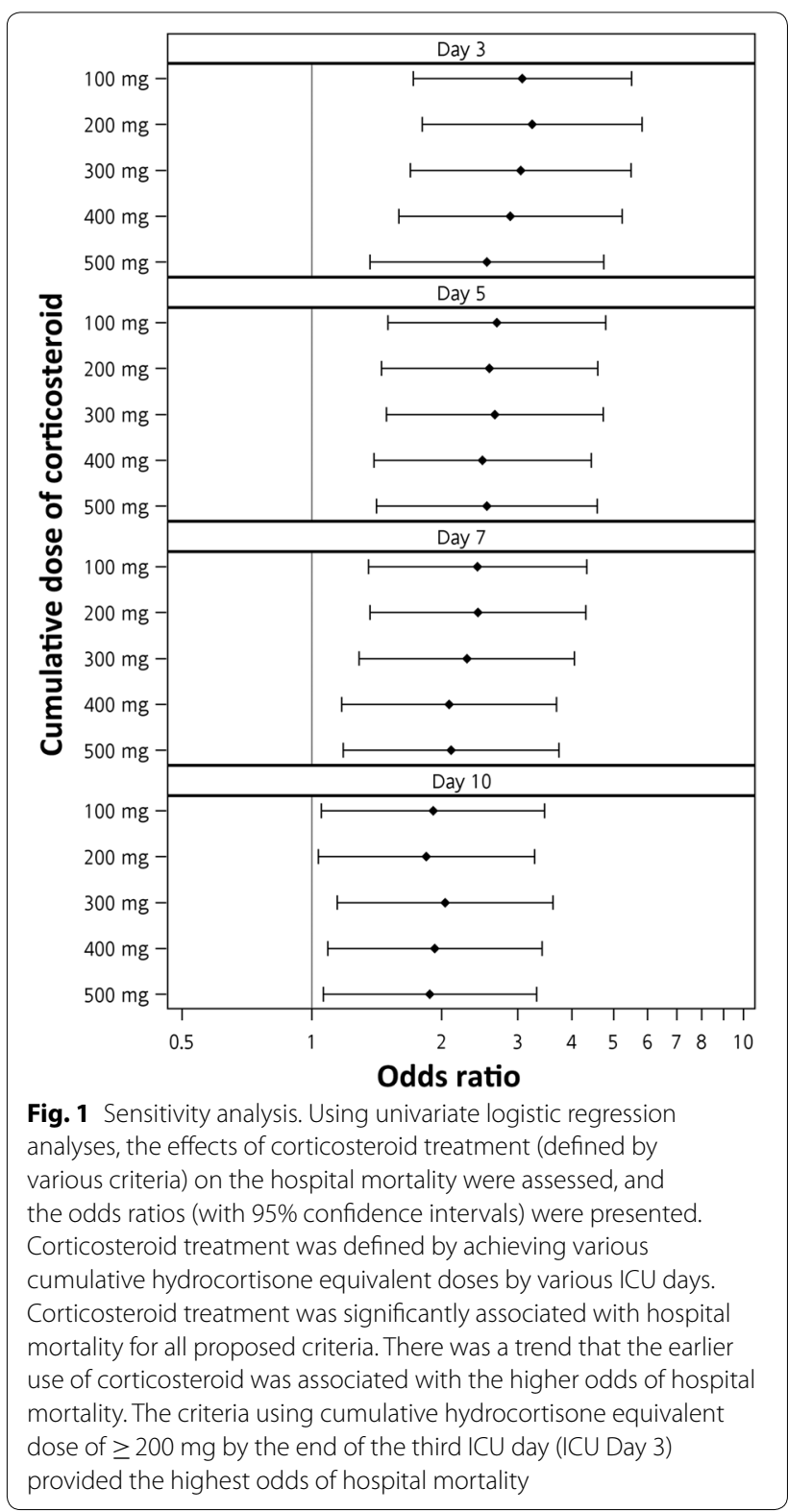


influenza-associated ARDS. The criteria using cumulative hydrocortisone equivalent dose of $\geq 200 \mathrm{mg}$ by the end of the third ICU day provided the highest odds of hospital mortality in the univariate logistic regression analysis, and was therefore used as the definition for "early corticosteroid treatment" in the subsequent analyses.

Using this definition, the study population was divided into 85 patients received early corticosteroid treatment and 156 patients without early corticosteroid treatment (Table 1). The patients received early corticosteroid treatment had higher hospital mortality rate than those without early corticosteroid treatment [43.5\% (37/85) vs. $19.2 \%(30 / 156), p<0.001]$. The patients receiving early corticosteroid treatment also had lower probability of survival than those without early corticosteroid treatment (log rank $p<0.001$ ) (Fig. 2). Multivariable logistic regression analysis constructed with a stepwise variable selection method showed that early corticosteroid treatment remained an independent risk factor for hospital mortality [adjusted OR (95\% CI) $=5.02$ (2.39-10.54), $p<0.001$ ] after adjusting for APACHE II score, underlying malignancy, influenza type, and ECMO treatment (Table 2). On subgroup analyses, early corticosteroid treatment remained an independent risk factor for hospital mortality in nearly all subgroups (Fig. 3). In the Cox proportional hazards regression analysis adjusting for APACHE II score, underlying malignancy, influenza type, and ECMO treatment, early corticosteroid treatment remained an independent risk factor for mortality [adjusted HR $(95 \% \mathrm{CI})=2.49(1.46-4.24), p<0.001$ ].

The multivariable logistic regression models were also used to investigate the effect of dosing and timing of corticosteroid treatment on hospital mortality. The odds of hospital mortality significantly increased by $9 \%(2-16 \%)$, $6 \%(2-11 \%)$, and $4 \%(1-8 \%)$ with every $100 \mathrm{mg}$ of cumulative hydrocortisone equivalent dose within 3, 5, 7 days after ICU admission, respectively (Additional file 1: Table S2), suggesting that earlier corticosteroid treatment and higher dosing were associated with higher hospital mortality. As shown in Additional file 1: Table S3, receiving corticosteroid treatment to a cumulative hydrocortisone equivalent dose reaching $\geq 200 \mathrm{mg}$ within 3 days after ICU admission increased the odds of hospital mortality [adjusted OR $(95 \% \mathrm{CI})=4.58 \quad(2.06-10.17)$, $p<0.001]$.

Because corticosteroid treatment might predispose patients to bacterial infections, we further analyzed the associations between corticosteroid treatment and subsequent ICU-acquired bacterial infections, defined as positive culture results from the samples collected after the third ICU day. Early corticosteroid treatment was associated with a significantly increased odds of subsequent bacteremia [adjusted OR $(95 \% \mathrm{CI})=2.37$ (1.01-5.56)] and a trend of increased odds of positive culture from any samples (Additional file 1: Table S4). However, the subsequent ICU-acquired bacteremia or positive culture results from any samples was not independently associated with increased odds of hospital mortality, whereas positive culture results from respiratory samples collected after the third ICU day was independently associated with increased odds of hospital mortality [adjusted OR $(95 \% \mathrm{CI})=2.20(1.01-4.81)$, $p=0.048$ ] (Additional file 1: Table S5).

In our study population, 154 patients (63.9\%) had ever received corticosteroid treatment at any dose within 14 days after ICU admission, including 104 survivors and 50 non-survivors (Additional file 1: Table S6). Compared with the survivors, the non-survivors had significantly shorter interval between ICU admission and the start of any corticosteroid treatment $(p=0.036)$ and a trend for longer duration of corticosteroid treatment $(p=0.054)$, whereas the total dose of corticosteroid used by the 14th day after ICU admission was similar in the survivors and non-survivors.

\section{Analyses of the propensity score-matched cohort}

To further confirm our findings, we built a propensity score-matched cohort, containing 85 patients with early corticosteroid treatment and 85 patients without early corticosteroid treatment (Additional file 1: Table S7). Both groups had similar clinical characteristics and received similar specific treatments. The patients with early corticosteroid treatment had a higher hospital mortality rate than those without early corticosteroid treatment $[43.5 \%(37 / 85)$ vs. $14.1 \%$ $(18 / 85), p<0.001]$. The patients with early corticosteroid treatment also had lower probability of survival than those without early corticosteroid treatment (log rank $p=0.004$ ) (Additional file 1: Fig. S3). Multivariable logistic regression analysis constructed with a stepwise variable selection method showed that early corticosteroid treatment remained an independent risk factor for hospital mortality [adjusted OR (95\% CI) $=4.30$ (1.90-9.75), $p<0.001]$ after adjusting for APACHE II score and ECMO treatment (Additional file 1: Table S8). On subgroup analyses, early corticosteroid treatment remained an independent risk factor for hospital mortality in nearly all subgroups (Additional file 1: Fig. S4). In the Cox proportional hazards regression analysis adjusting for APACHE II score and ECMO treatment, early corticosteroid treatment remained an independent risk factor for mortality [adjusted HR $(95 \% \mathrm{CI})=2.62(1.46-4.70), p=0.001]$. 
Table 1 Characteristics and outcomes between influenza-associated ARDS patients with versus without early corticosteroid treatment

\begin{tabular}{|c|c|c|c|}
\hline Variables & Early $\mathrm{CS}$ treatment $(\mathrm{N}=85)$ & No early CS treatment $(\mathrm{N}=156)$ & $p$ value \\
\hline Age & $61.0(56.0-68.0)$ & $59.0(49.0-65.5)$ & 0.211 \\
\hline Male gender & $52(61.2)$ & $101(64.7)$ & 0.674 \\
\hline APACHE II score & $24.0(18.0-30.0)$ & $23.0(17.0-29.0)$ & 0.129 \\
\hline $\mathrm{BMl}$ & $24.6(22.1-28.1)$ & $24.8(21.2-28.1)$ & 0.808 \\
\hline \multicolumn{4}{|l|}{ Comorbidity } \\
\hline Malignancy & $7(8.2)$ & $22(14.1)$ & 0.217 \\
\hline Diabetes & $26(30.6)$ & $43(27.6)$ & 0.655 \\
\hline Cerebrovascular disease & $8(9.4)$ & $10(6.4)$ & 0.445 \\
\hline Chronic airway disease & $10(11.8)$ & $11(7.1)$ & 0.237 \\
\hline End-stage renal disease & 7 (8.2) & $7(4.5)$ & 0.257 \\
\hline Congestive heart failure & $11(12.9)$ & $15(9.6)$ & 0.515 \\
\hline Influenza & & & 0.539 \\
\hline Type A & $61(71.8)$ & $118(75.6)$ & \\
\hline Type B & $24(28.2)$ & $38(24.4)$ & \\
\hline $\mathrm{PaO}_{2} / \mathrm{FiO}_{2}$ ratio & $87.5(56.0-142.3)$ & $84.0(62.0-149.6)$ & 0.670 \\
\hline Severity of ARDS & & & 0.539 \\
\hline Severe ARDS & $46(54.1)$ & $95(60.9)$ & \\
\hline Moderate ARDS & $32(37.6)$ & $41(26.3)$ & \\
\hline Mild ARDS & 7 (8.2) & $20(12.8)$ & \\
\hline \multicolumn{4}{|l|}{ Laboratory data } \\
\hline WBC count, $\times 10^{3} / \mu \mathrm{L}$ & $9.6(5.5-15.5)$ & $8.8(5.9-13.0)$ & 0.253 \\
\hline Hemoglobin, g/dL & $12.5(10.5-14.2)$ & $11.9(10.0-13.5)$ & 0.102 \\
\hline Platelet count, $\times 10^{3} / \mu \mathrm{L}$ & $150.0(108.5-200.5)$ & $149.0(102.0-210.0)$ & 0.770 \\
\hline Albumin, $\mathrm{g} / \mathrm{dL}$ & $2.9(2.5-3.3)$ & $2.9(2.5-3.2)$ & 0.670 \\
\hline C-reactive protein, $\mathrm{mg} / \mathrm{dL}$ & $14.1(4.2-23.3)$ & $14.9(6.7-23.0)$ & 0.727 \\
\hline Bacterial coinfections $^{\mathrm{a}}$ & $14(16.5)$ & $17(10.9)$ & 0.231 \\
\hline \multicolumn{4}{|l|}{ Specific treatment } \\
\hline Mechanical ventilation & $85(100.0)$ & $156(100.0)$ & 0.539 \\
\hline Prone positioning or ECMO & $36(42.4)$ & $56(35.9)$ & 0.334 \\
\hline Prone positioning & $29(34.1)$ & $29(18.6)$ & 0.011 \\
\hline ECMO & $11(12.9)$ & $30(19.2)$ & 0.281 \\
\hline Vasopressor infusion & $49(57.6)$ & $72(46.2)$ & 0.105 \\
\hline Hemodialysis & $16(18.8)$ & $24(15.4)$ & 0.587 \\
\hline \multicolumn{4}{|l|}{ Clinical outcomes } \\
\hline Hospital mortality & $37(43.5)$ & $30(19.2)$ & $<0.001$ \\
\hline Hospital days in survivors & $24.8(17.1-40.1)$ & $28.2(19.1-44.4)$ & 0.475 \\
\hline ICU days in survivors & $13.8(8.4-23.0)$ & $14.8(9.1-21.8)$ & 0.664 \\
\hline Ventilator days in survivors & $11.9(8.4-25.3)$ & $14.4(7.8-21.7)$ & 0.953 \\
\hline ICU-free days at Day 28 & $0.0(0.0-15.2)$ & $9.2(0.0-17.6)$ & 0.012 \\
\hline Ventilator-free days at Day 28 & $0.0(0.0-17.1)$ & $11.0(0.0-19.6)$ & 0.009 \\
\hline
\end{tabular}

APACHE II Acute Physiology and Chronic Health Evaluation II, ARDS acute respiratory distress syndrome, BMI body mass index, CS corticosteroid, ECMO extracorporeal membrane oxygenation, $\mathrm{FiO}_{2}$ fraction of inspired oxygen, $\mathrm{PaO}_{2}$ partial pressure of arterial oxygen, WBC white blood cell

Statistics are presented as the median (25th-75th percentiles) for continuous variables and as number (\%) for categorical variables, as appropriate. $p$ values are calculated by Mann-Whitney $U$ test (or known as Wilcoxon rank sum test) and Chi-square test for continuous and categorical variables, respectively

${ }^{a}$ Bacterial coinfection was defined as positive bacterial cultures from blood, pleural effusion, lower respiratory tract secretion, or urine samples within $48 \mathrm{~h}$ of ARDS diagnosis 


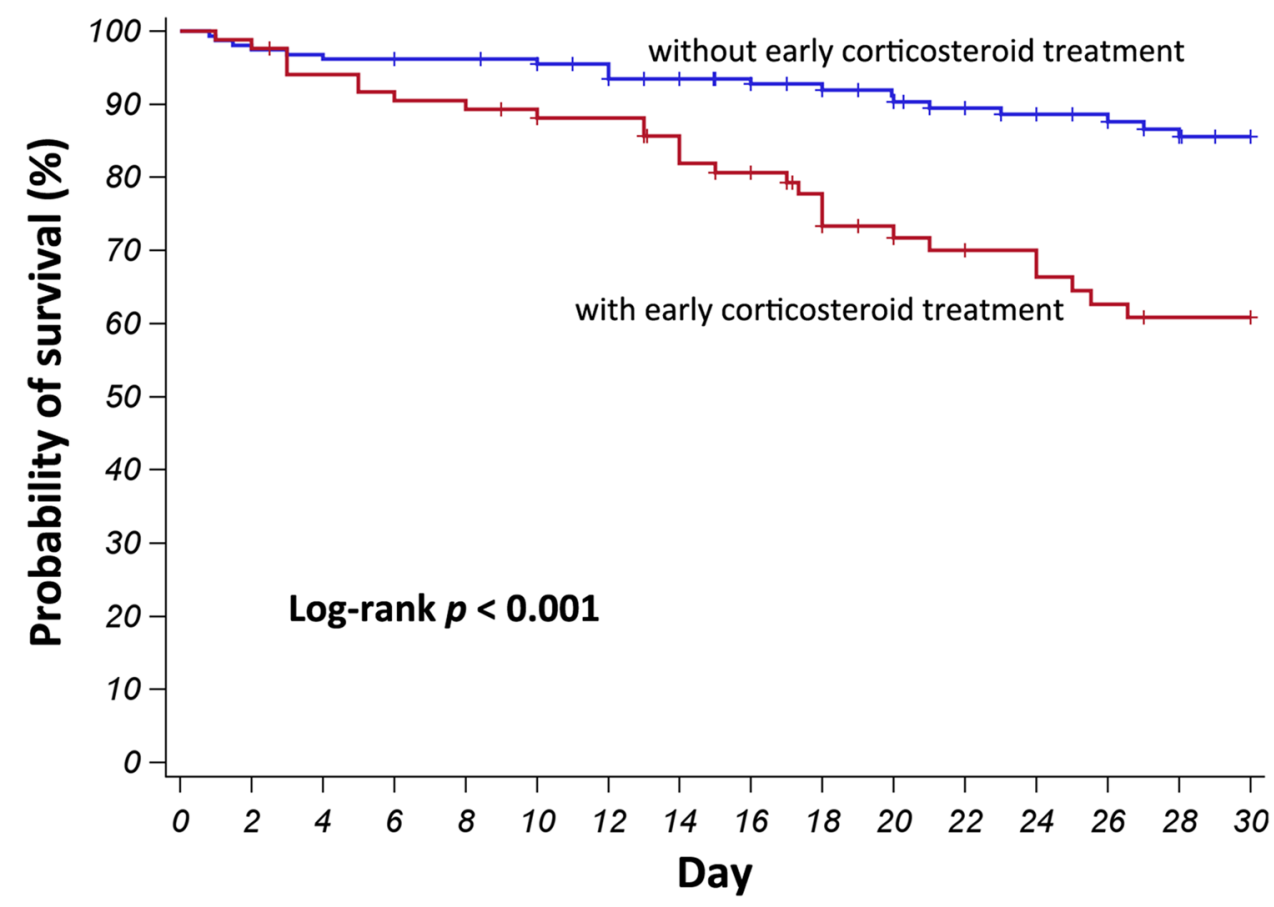

No. at risk

$\begin{array}{lllllllllllllllll}\text { No earlyCS } & 156 & 152 & 151 & 149 & 149 & 147 & 140 & 135 & 124 & 116 & 110 & 102 & 97 & 88 & 76 & 68\end{array}$

$\begin{array}{lllllllllllllllll}\text { Early CS } & 85 & 83 & 79 & 77 & 75 & 74 & 72 & 65 & 60 & 51 & 43 & 40 & 36 & 34 & 32 & 31\end{array}$

Fig. 2 Probability of survival from ICU admission to Day 30. The patients were classified by whether they received early corticosteroid (CS) treatment or not

Table 2 Univariate and multivariable analyses of factors associated with hospital mortality in patients with influenzaassociated ARDS

\begin{tabular}{|c|c|c|c|c|}
\hline \multirow[t]{2}{*}{ Variables } & \multicolumn{2}{|c|}{ Univariate analysis $^{a}$} & \multicolumn{2}{|l|}{ Multivariable analysis $^{\dagger}$} \\
\hline & OR $(95 \% \mathrm{Cl})$ & $p$ value & Adjusted OR (95\% Cl) & $p$ value \\
\hline APACHE II score & $1.11(1.07-1.16)$ & $<0.001$ & $1.12(1.07-1.17)$ & $<0.001$ \\
\hline $\mathrm{PaO}_{2} / \mathrm{FiO}_{2}$ ratio & $0.99(0.99-1.00)$ & 0.007 & & \\
\hline WBC & $1.00(1.00-1.00)$ & 0.015 & & \\
\hline Albumin, $\mathrm{g} / \mathrm{dL}$ & $0.55(0.31-0.97)$ & 0.039 & & \\
\hline Malignancy & $2.80(1.27-6.18)$ & 0.011 & $2.71(1.06-6.90)$ & 0.037 \\
\hline Influenza type A & $0.50(0.27-0.93)$ & 0.028 & $0.38(0.18-0.82)$ & 0.013 \\
\hline ECMO & $4.53(2.25-9.14)$ & $<0.001$ & $8.51(3.52-20.55)$ & $<0.001$ \\
\hline Vasopressor infusion & $2.89(1.59-5.25)$ & 0.001 & & \\
\hline Hemodialysis & $2.54(1.26-5.12)$ & 0.009 & & \\
\hline Early CS treatment & $3.24(1.80-5.81)$ & $<0.001$ & $5.02(2.39-10.54)$ & $<0.001$ \\
\hline
\end{tabular}

APACHE II Acute Physiology and Chronic Health Evaluation II, ARDS acute respiratory distress syndrome, $B M I$ body mass index, $C l$ confidence interval, $C S$ corticosteroid, $E C M O$ extracorporeal membrane oxygenation, $\mathrm{FiO}_{2}$ fraction of inspired oxygen, $\mathrm{PaO}_{2}$ partial pressure of arterial oxygen, $\mathrm{OR}$ odds ratio, $W B C$ white blood cell

a The variable representing early CS treatment, basic demographic variables, and all clinical variables possibly associated with hospital mortality were analyzed in univariate logistic regression models

+ We replaced missing values (APACHE II score in 4 patients, WBC count in 2 patients, and albumin in 40 patients) by the corresponding overall median values for the multivariable regression analysis. Variables associated with hospital mortality with a $p$ value $<0.05$ in univariate models were selected into the multivariable logistic regression model, using a stepwise algorithm with criteria of $p>0.05$ for eliminating variables 


\begin{tabular}{|c|c|c|c|c|c|}
\hline \multirow[t]{2}{*}{ Subgroup } & \multicolumn{2}{|c|}{ Early CS Use } & \multirow[t]{2}{*}{ Forest Plot for Odds Ratio } & \multicolumn{2}{|c|}{ Odds Ratio $(95 \% \mathrm{Cl})$} \\
\hline & Yes & No & & & \\
\hline \multicolumn{6}{|l|}{ Age } \\
\hline$<60$ yrs & 38 & 79 & $\longrightarrow$ & 5.48 & $(1.89-15.92)$ \\
\hline$\geq 60 \mathrm{yrs}$ & 47 & 77 & & 5.49 & $(1.81-16.70)$ \\
\hline \multicolumn{6}{|l|}{ Gender } \\
\hline Male & 52 & 101 & $\longrightarrow$ & 3.46 & $(1.42-8.47)$ \\
\hline Female & 33 & 55 & & 9.01 & $(2.20-36.96)$ \\
\hline \multicolumn{6}{|l|}{ APACHE II Score } \\
\hline$<24$ & 34 & 75 & $\longrightarrow$ & 6.22 & $(1.42-27.28)$ \\
\hline$\geq 24$ & 51 & 81 & $\longrightarrow$ & 4.24 & $(1.84-9.76)$ \\
\hline \multicolumn{6}{|l|}{ Influenza Type } \\
\hline A & 61 & 118 & $\longrightarrow$ & 3.59 & $(1.49-8.63)$ \\
\hline B & 24 & 38 & - & 12.15 & $(2.77-53.26)$ \\
\hline \multicolumn{6}{|l|}{ ARDS Severity } \\
\hline Severe & 46 & 95 & $\longrightarrow$ & 4.52 & $(1.76-11.59)$ \\
\hline Mild-Moderate & 39 & 61 & - & 10.34 & $(2.33-45.90)$ \\
\hline \multicolumn{6}{|c|}{ Bacterial Coinfection } \\
\hline Yes & 39 & 61 & - & 10.34 & $(2.33-45.90)$ \\
\hline No & 85 & 156 & - & 5.56 & $(2.59-11.94)$ \\
\hline \multicolumn{6}{|c|}{ Vasopressor infusion } \\
\hline Yes & 49 & 72 & - & 5.88 & $(2.13-16.29)$ \\
\hline No & 36 & 84 & - & 4.12 & $(1.27-13.44)$ \\
\hline \multicolumn{6}{|l|}{ Prone } \\
\hline Yes & 29 & 29 & & 11.81 & $(1.03-134.94)$ \\
\hline No & 56 & 127 & $\longrightarrow$ & 5.00 & $(2.07-12.05)$ \\
\hline \multicolumn{6}{|l|}{ ECMO } \\
\hline Yes & 11 & 30 & - & 10.88 & $(1.08-110.10)$ \\
\hline No & 74 & 126 & $\longrightarrow$ & 4.51 & $(2.02-10.08)$ \\
\hline \multicolumn{6}{|c|}{ Chronic Airway Disease } \\
\hline Yes & 10 & 11 & + & & \\
\hline No & 75 & 145 & $\longrightarrow$ & 4.98 & $(2.29-10.82)$ \\
\hline \multirow[t]{3}{*}{ Overall } & 85 & 156 & $\longrightarrow$ & 5.02 & $(2.39-10.54)$ \\
\hline & & $T$ & 1 & $1 \quad 1$ & \\
\hline & & 0.1 & $\begin{array}{lllll}1 & 2 & 3 & 5 & 10\end{array}$ & 100200 & \\
\hline
\end{tabular}

\section{Discussion}

In this multicenter cohort study, we found that a substantial proportion of patients with influenza-associated ARDS had received corticosteroid treatment and more than a third of them received $\geq 200 \mathrm{mg}$ hydrocortisone equivalent dose within 3 days after ICU admission, although some previous literatures did not support this treatment. Higher APACHE II score, underlying malignancy, influenza type B, ECMO, and early corticosteroid treatment were significantly associated with hospital mortality in patients with influenza-associated ARDS.
The risk of hospital mortality increased with the earlier use of corticosteroid treatment and with the higher cumulative dose of corticosteroid treatment. The analyses of a propensity score-matched cohort showed consistent results.

During the 2009 pandemic period, the World Health Organization published revised guidelines for the pharmacological management of pandemic influenza A (H1N1) [21]. Although "patients who have severe or progressive clinical illness, including viral pneumonitis, respiratory failure, and ARDS due to influenza virus 
infection, should not be given systemic corticosteroids unless indicated for other reasons or as part of an approved research protocol" have been clearly stated, the adherence to this suggestion remained poor [2, 21]. In the Chinese study collecting 613 patients of avian influenza A (H7N9) viral pneumonia between April 2013 to March 2015, as much as $70.8 \%$ of patients received corticosteroids [9]. Similarly, in our study, $57.7 \%$ of patients had received $\geq 200 \mathrm{mg}$ hydrocortisone equivalent dose within 2 weeks after ICU admission and $63.9 \%$ of patients had ever received corticosteroid treatment at any dose during this period.

Many studies have investigated the role of systemic adjunctive corticosteroid treatment in pneumonia. A systematic review and meta-analysis including 13 RCTs showed that systemic corticosteroid treatment significantly decreased the need for mechanical ventilation and the risk of incident ARDS in adult patients hospitalized with severe community-acquired pneumonia (CAP), while a significantly decreased mortality was only found in those with severe pneumonia [22]. A large doubleblind, multicenter, randomized, placebo-controlled study also showed prednisone treatment for 7 days shortened the time to clinical stability in patients admitted with CAP [23].

The excessive production of pro-inflammatory cytokines in sepsis may lead to relative adrenal insufficiency and/or peripheral glucocorticoid resistance [24]. Corticosteroid treatment decreases pro-inflammatory mediators and improves innate immunity $[25,26]$. A multicenter RCT showed that low-dose hydrocortisone and fludrocortisone significantly reduced the mortality in patients with septic shock [27]. Another multicenter RCT of septic shock patients showed that hydrocortisone treatment hastened the reversal of shock, but did not improve survival [28]. Two large RCTs, APROCCHSS and ADRENAL, both showed the effect of corticosteroid treatment in enhancing shock reversal and decreasing the duration of mechanical ventilation in patients with septic shock [29, 30]. APROCCHSS showed that hydrocortisone plus fludrocortisone treatment decreased the 90-day mortality, whereas ADRENAL showed no significant difference in 90-day mortality in patients receiving hydrocortisone or those receiving placebo $[29,30]$. Our study showed that shock (vasopressor infusion) was associated with significantly increased hospital mortality. However, the need of vasopressor infusion was excluded during stepwise variable selection for composing the multivariable model, suggesting that corticosteroid treatment might be a greater predictor for hospital mortality. In addition, the results of subgroup analysis showed that early corticosteroid was associated with increased hospital mortality in both vasopressor group and no vasopressor group, suggesting this association was independent of septic shock.

The underlying mechanisms why corticosteroid treatment increased mortality in influenza-associated ARDS remained unclear. In a large RCT of septic shock, patients treated with hydrocortisone therapy had more episodes of superinfections than those receiving placebo [28]. However, the Canadian study of 2009 pandemic influenza A (H1N1)-related critical illness showed no significant difference in nosocomial (respiratory and bloodstream) infections between the patients receiving corticosteroids and the others [8]. Our current study showed that early corticosteroid treatment might increase the risk of subsequent bacteremia, whereas the isolation of bacteria from respiratory samples was associated with increased hospital mortality.

Our study has several strengths. Firstly, our study enrolled patients from multiple medical centers across Taiwan, providing a great opportunity to investigate the real-world circumstance. Designing and conducting an RCT for pandemic critical illness is never easy [31]. To the best of our knowledge, no RCT discussing the role of corticosteroid treatment in influenza-associated ARDS has been done, and our study is one of the largest cohort studies focusing on this topic to date. Secondly, we only included patients receiving invasive mechanical ventilation for influenza-associated ARDS. In contrast to the previous studies, which included either patients with milder influenza or patients with ARDS from various etiologies, our homogenous cohort provided us a better opportunity for investigating the effect of corticosteroid treatment in the patients with influenza-associated ARDS [1, 2, 5, 9-11]. Thirdly, we performed thorough analyses, using variable timing and dosing definitions for corticosteroid treatment. These analyses not only showed consistent results, but also showed the dose-dependent and timing-related effects of corticosteroid treatment.

Nonetheless, our study still has limitations. Firstly, the retrospective nature of our study resulted in few missing values despite the effort in data collection, and might also bring some bias, such as confounding by indications of corticosteroid treatment (i.e., corticosteroid treatment might be used more frequently and earlier in sicker patients). A hard outcome of hospital mortality was therefore adopted. In a Canadian study of influenza-associated critical illness, the use of corticosteroid treatment was mainly associated with hemodynamic instability and chronic airway disease [32]. The subgroup analyses, classifying patients by vasopressor infusion or chronic airway disease, showed consistent results in our study. We also performed multivariable analyses to adjust all possible confounders. To account for residual confounding by indication, we performed another set of 
analyses in a propensity score-matched sub-cohort, with increased comparability between groups, and found consistent results. Nevertheless, despite efforts to mitigate the effects of residual confounding factors, this potential problem could not be totally solved. The results of this study must therefore be interpreted with caution. Further RCTs or well-designed prospective studies are warranted to understand the benefits or harms associated with corticosteroid treatment in patients with influenzaassociated ARDS. Secondly, the effects of different types of corticosteroids were not investigated in the current study because some patients used more than one type of corticosteroid and our study had insufficient power to investigate this aspect. To investigate the effect of corticosteroids, we transfer all corticosteroids into hydrocortisone equivalent dose. Although we believe our finding is a class effect of corticosteroids, the differences between various corticosteroids need to be investigated in a larger study in the future.

\section{Conclusions}

The current study showed that early corticosteroid treatment was associated with a significantly higher hospital mortality in adult patients with influenza-associated ARDS. The earlier the treatment, the higher the mortality. The higher the corticosteroid dose, the higher the mortality. While further RCTs or well-designed prospective studies are warranted to confirm our findings, we suggest that clinicians should be cautious about using corticosteroid treatment in this patient group.

\section{Supplementary information}

Supplementary information accompanies this paper at https://doi. org/10.1186/s13613-020-0642-4.

Additional file 1. Additional tables and figures.

\begin{abstract}
Abbreviations
APACHE II: Acute Physiology and Chronic Health Evaluation II; ARDS: Acute res piratory distress syndrome; $\mathrm{BMl}$ : Body mass index; $\mathrm{Cl}$ : Confidence interval; CS: Corticosteroid; ECMO: Extracorporeal membrane oxygenation; $\mathrm{FiO}_{2}$ : Fraction of inspired oxygen; HR: Hazard ratio; ICU: Intensive care unit; OR: Odds ratio; $\mathrm{PaO}_{2}$ : Partial pressure of arterial oxygen; RCT: Randomized controlled study; WBC: White blood cell.
\end{abstract}

\section{Acknowledgements}

We thank Tse-Kuang Kai for the assistance in statistical analysis. We thank Professor Chong-Jen Yu, the former President of the Taiwan Society of Pulmonary and Critical Care Medicine, and Professor Meng-Chih Lin, the current President of the Taiwan Society of Pulmonary and Critical Care Medicine, who organized and coached the TSIRC team.

\section{Authors' contributions}

KYY, MCC, KCK, HCW, WCP, CLW, SJL, WFF, and CCS had the idea of the study and made substantial contributions to conception and design. MJT, KYY, MCC, KCK, HCW, WCP, CLW, SJL, WFF, and CCS made substantial contributions to acquisition of data. MJT and CCS made substantial contributions to the analysis and interpretation of data. MJT, WAC, JRT, and CCS drafted the manuscript. All authors read and approved the final manuscript.

\section{Funding}

This study was partially supported by Chang Gung Medical Foundation CMRPG3F0791, MOST research project Grants MOST-105-2314-B-010-041-MY3, and Taipei Veterans General Hospital Grants V107C-077.

\section{Availability of data and materials \\ Not applicable.}

\section{Ethics approval and consent to participate}

This study was approved by the Institutional Review Board in each participating hospital, including Taichung Veterans General Hospital (CE16093A), National Taiwan University Hospital (201605036RIND), Taipei Veterans General Hospital (2016-05-020CC), Tri-Service General Hospital (1-105-05-086), Chang-Gung Memorial Hospital (201600988B0), China Medical University Hospital (105-REC2-053(FR)), Kaohsiung Medical University Hospital (KUMHIRB-E(I)-20170097), and Kaohsiung Chang-Gung Memorial Hospital (201600988B0). No consent was required for this type of study.

\section{Consent for publication}

Not applicable.

\section{Competing interests}

On behalf of all authors, the corresponding author states that there is no conflict of interest.

\section{Author details}

${ }^{1}$ Division of Pulmonary and Critical Care Medicine, Department of Internal Medicine, Kaohsiung Medical University Hospital, Kaohsiung Medical University, No. 100, Tz-You 1st Road, Kaohsiung 807, Taiwan. ${ }^{2}$ Department of Internal Medicine, School of Medicine, College of Medicine, Kaohsiung Medical University, Kaohsiung, Taiwan. ${ }^{3}$ Graduate Institute of Clinical Medicine, College of Medicine, Kaohsiung Medical University, Kaohsiung, Taiwan. ${ }^{4}$ Department of Respiratory Therapy, College of Medicine, Kaohsiung Medical University, Kaohsiung, Taiwan. ${ }^{5}$ Department of Chest Medicine, Taipei Veterans General Hospital, Taipei, Taiwan. ${ }^{6}$ Institute of Emergency and Critical Care Medicine, School of Medicine, National Yang-Ming University, Taipei, Taiwan. ${ }^{7}$ Division of Chest Medicine, Department of Internal Medicine, Taichung Veterans General Hospital, Taichung, Taiwan. ${ }^{8}$ Central Taiwan University of Science and Technology, Taichung, Taiwan. ${ }^{9}$ Tunghai University, Taichung, Taiwan. ${ }^{10}$ Department of Thoracic Medicine, Chang Gung Memorial Hospital, Taoyuan, Taiwan. ${ }^{11}$ Department of Respiratory Therapy, Chang-Gung University College of Medicine, Taoyuan, Taiwan. ${ }^{12}$ Division of Chest Medicine, Department of Internal Medicine, National Taiwan University Hospital, Taipei, Taiwan. ${ }^{13}$ Division of Pulmonary and Critical Care Medicine, Department of Internal Medicine, Tri-Service General Hospital, National Defense Medical Center, Taipei, Taiwan. ${ }^{14}$ Center for Quality Management, Taichung Veterans General Hospital, Taichung, Taiwan. ${ }^{15}$ Division of Pulmonary and Critical Care, Department of Internal Medicine, China Medical University Hospital, Taichung, Taiwan. ${ }^{16}$ Division of Pulmonary and Critical Care Medicine, Department of Internal Medicine, Kaohsiung Chang Gung Memorial Hospital, Kaohsiung, Taiwan. ${ }^{17}$ Department of Respiratory Care, Chang Gung University of Science and Technology, Chiayi, Taiwan. ${ }^{18}$ Faculty of Medicine, School of Medicine, National Yang-Ming University, Taipei, Taiwan. ${ }^{19}$ Department of Medical Research, Taichung Veterans General Hospital, Taichung, Taiwan.

Received: 25 November 2019 Accepted: 18 February 2020 Published online: 27 February 2020

\section{References}

1. Lansbury L, Rodrigo C, Leonardi-Bee J, Nguyen-Van-Tam J, Lim WS. Corticosteroids as adjunctive therapy in the treatment of influenza. Cochrane Database Syst Rev. 2019;2:CD010406.

2. Rodrigo C, Leonardi-Bee J, Nguyen-Van-Tam JS, Lim WS. Effect of corticosteroid therapy on influenza-related mortality: a systematic review and meta-analysis. J Infect Dis. 2015;212(2):183-94. 
3. Chow EJ, Doyle JD, Uyeki TM. Influenza virus-related critical illness: prevention, diagnosis, treatment. Crit Care. 2019;23(1):214.

4. de Jong MD, Simmons CP, Thanh TT, Hien VM, Smith GJ, Chau TN, et al. Fatal outcome of human influenza A (H5N1) is associated with high viral load and hypercytokinemia. Nat Med. 2006;12(10):1203-7.

5. Ruan SY, Lin HH, Huang CT, Kuo PH, Wu HD, Yu CJ. Exploring the heterogeneity of effects of corticosteroids on acute respiratory distress syndrome: a systematic review and meta-analysis. Crit Care. 2014;18(2):R63.

6. Beigel JH, Farrar J, Han AM, Hayden FG, Hyer R, de Jong MD, et al. Avian influenza A (H5N1) infection in humans. N Engl J Med. 2005;353(13):1374-85.

7. Brun-Buisson C, Richard JC, Mercat A, Thiebaut AC, Brochard L, Group R-SAHNvR. Early corticosteroids in severe influenza A/H1N1 pneumonia and acute respiratory distress syndrome. Am J Respir Crit Care Med. 2011;183(9):1200-6.

8. Delaney JW, Pinto R, Long J, Lamontagne F, Adhikari NK, Kumar A, et al. The influence of corticosteroid treatment on the outcome of influenza A(H1N1 pdm09)-related critical illness. Crit Care. 2016:20:75.

9. Cao B, Gao H, Zhou B, Deng X, Hu C, Deng C, et al. Adjuvant corticosteroid treatment in adults with influenza A (H7N9) viral pneumonia. Crit Care Med. 2016;44(6):e318-28.

10. Zhang Y, Sun W, Svendsen ER, Tang S, Maclntyre RC, Yang P, et al. Do corticosteroids reduce the mortality of influenza A ( $\mathrm{H} 1 \mathrm{~N} 1)$ infection? A meta-analysis. Crit Care. 2015;19:46.

11. Moreno G, Rodriguez A, Reyes LF, Gomez J, Sole-Violan J, Diaz E, et al. Corticosteroid treatment in critically ill patients with severe influenza pneumonia: a propensity score matching study. Intensive Care Med. 2018;44(12):2319-20.

12. Sweeney RM, McAuley DF. Acute respiratory distress syndrome. Lancet. 2016;388(10058):2416-30.

13. Munshi L, Rubenfeld G, Wunsch H. Adjuvants to mechanical ventilation for acute respiratory distress syndrome. Intensive Care Med. 2016:42(5):775-8.

14. Tang BM, Craig JC, Eslick GD, Seppelt I, McLean AS. Use of corticosteroids in acute lung injury and acute respiratory distress syndrome: a systematic review and meta-analysis. Crit Care Med. 2009;37(5):1594-603.

15. Steinberg KP, Hudson LD, Goodman RB, Hough CL, Lanken PN, Hyzy R, et al. Efficacy and safety of corticosteroids for persistent acute respiratory distress syndrome. N Engl J Med. 2006;354(16):1671-84.

16. Bellani G, Laffey JG, Pham T, Fan E, Brochard L, Esteban A, et al. Epidemiology, patterns of care, and mortality for patients with acute respiratory distress syndrome in intensive care units in 50 countries. JAMA. 2016;315(8):788-800.

17. Chan MC, Chao WC, Liang SJ, Tseng CH, Wang HC, Chien YC, et al. First tidal volume greater than $8 \mathrm{~mL} / \mathrm{kg}$ is associated with increased mortality in complicated influenza infection with acute respiratory distress syndrome. J Formos Med Assoc. 2018;118(1 Pt 2):378-85.

18. Kao KC, Chang KW, Chan MC, Liang SJ, Chien YC, Hu HC, et al. Predictors of survival in patients with influenza pneumonia-related severe acute respiratory distress syndrome treated with prone positioning. Ann Intensive Care. 2018:8(1):94
19. Chao WC, Tseng CH, Chien YC, Sheu CC, Tsai MJ, Fang WF, et al. Association of day 4 cumulative fluid balance with mortality in critically ill patients with influenza: a multicenter retrospective cohort study in Taiwan. PLoS ONE. 2018;13(1):e0190952.

20. Force ADT, Ranieri VM, Rubenfeld GD, Thompson BT, Ferguson ND, Caldwell $E$, et al. Acute respiratory distress syndrome: the Berlin definition. JAMA. 2012;307(23):2526-33.

21. WHO. WHO guidelines for the pharmacological management of pandemic influenza A(H1N1) 2009 and other influenza viruses. Revised February 2010. Part I recommendations. Geneva: WHO; 2010.

22. Siemieniuk RA, Meade MO, Alonso-Coello P, Briel M, Evaniew N, Prasad M, et al. Corticosteroid therapy for patients hospitalized with communityacquired pneumonia: a systematic review and meta-analysis. Ann Intern Med. 2015;163(7):519-28.

23. Blum CA, Nigro N, Briel M, Schuetz P, Ullmer E, Suter-Widmer I, et al. Adjunct prednisone therapy for patients with community-acquired pneumonia: a multicentre, double-blind, randomised, placebo-controlled trial. Lancet. 2015;385(9977):1511-8.

24. Prigent H, Maxime V, Annane D. Clinical review: corticotherapy in sepsis. Crit Care. 2004;8(2):122-9.

25. Kaufmann I, Briegel J, Schliephake F, Hoelzl A, Chouker A, Hummel T, et al. Stress doses of hydrocortisone in septic shock: beneficial effects on opsonization-dependent neutrophil functions. Intensive Care Med. 2008:34(2):344-9.

26. Rhen T, Cidlowski JA. Antiinflammatory action of glucocorticoids-new mechanisms for old drugs. N Engl J Med. 2005;353(16):1711-23.

27. Annane D, Sebille V, Charpentier C, Bollaert PE, Francois B, Korach $J M$, et al. Effect of treatment with low doses of hydrocortisone and fludrocortisone on mortality in patients with septic shock. JAMA. 2002;288(7):862-71.

28. Sprung CL, Annane D, Keh D, Moreno R, Singer M, Freivogel K, et al. Hydrocortisone therapy for patients with septic shock. N Engl J Med. 2008:358(2):111-24.

29. Annane D, Renault A, Brun-Buisson C, Megarbane B, Quenot JP, Siami S, et al. Hydrocortisone plus fludrocortisone for adults with septic shock. N Engl J Med. 2018;378(9):809-18.

30. Venkatesh B, Finfer S, Cohen J, Rajbhandari D, Arabi Y, Bellomo R, et al. Adjunctive glucocorticoid therapy in patients with septic shock. N Engl J Med. 2018;378(9):797-808.

31. Annane D, Antona M, Lehmann B, Kedzia C, Chevret S, Investigators

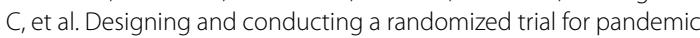
critical illness: the 2009 H1N1 influenza pandemic. Intensive Care Med. 2012;38(1):29-39.

32. Yale P, Adhikari NK, Masse V, Fowler RA, Xiong W, McGeer A, et al. Corticosteroid therapy in critical illness due to seasonal and pandemic influenza. Can Respir J. 2015;22(5):271-4.

\section{Publisher's Note}

Springer Nature remains neutral with regard to jurisdictional claims in published maps and institutional affiliations.

\section{Submit your manuscript to a SpringerOpen ${ }^{\odot}$ journal and benefit from:}

- Convenient online submission

- Rigorous peer review

- Open access: articles freely available online

- High visibility within the field

- Retaining the copyright to your article

Submit your next manuscript at $\boldsymbol{\nabla}$ springeropen.com 